\title{
Photon Upconversion in Aqueous Nanodroplets
}

\author{
Samuel N. Sanders, ${ }^{1}$ Mahesh K. Gangishetty, ${ }^{1}$ Matthew Y. Sfeir, ${ }^{2,3}$ Daniel \\ N. Congreve ${ }^{1, *}$
}

Supporting Information

\section{Spectroscopic Methods}

A commercial optical parametric amplifier (LightConversion) was used to generate excitation light. This excitation light was used to perform transient absorption spectroscopy using a commercial Ti:Sapphire laser system (SpectraPhysics $|800 \mathrm{~nm}| 100 \mathrm{fs}|3.5 \mathrm{~mJ}| 1 \mathrm{kHz}$ ). The probe light was an electronically synchronized supercontinuum (Leukos).

Photoluminescence spectra were excited using a Thorlabs laser diode as excitation source and collected using a fiber coupled Ocean Optics QE Pro spectrometer.

\section{Global Analysis}

Global (singular value decomposition-based) analysis was performed with the Glotaran software package (http://glotaran.org). ${ }^{1}$ These methods yield especially accurate fits of rate constants because they treat the entire data set in aggregate.

\section{Chemicals}

All commercially obtained reagents/solvents were used as received; chemicals were purchased from Alfa Aesar, Sigma-Aldrich, Acros organics, TCl America, Mallinckrodt, Gibco, Oakwood Product and Frontier Scientific and were used as received without further purification. The one exception is Tips Tetracene, which was synthesized according to literature. ${ }^{2}$

\section{Dynamic Light Scattering}

Dynamic light scattering was performed at room temperature in water on a Beckman Coulter Delsa Nano instrument. 


\section{Absorption and Photoluminescence}

As seen in Figure S1, most samples exhibited relatively subtle differences in absorption spectra going from dilute chloroform solution to swelled micelle or even unswelled micelle. The most noteworthy exception is BPEA, where the unswelled micelle spectrum has a significantly perturbed spectrum relative to the isolated molecules in solution. In contrast, the swelled micelles and the dilute BPEA in chloroform reveal similar spectra. This result suggests the BPEA micelles without a swelling agent suffer significantly from annihilator aggregation.
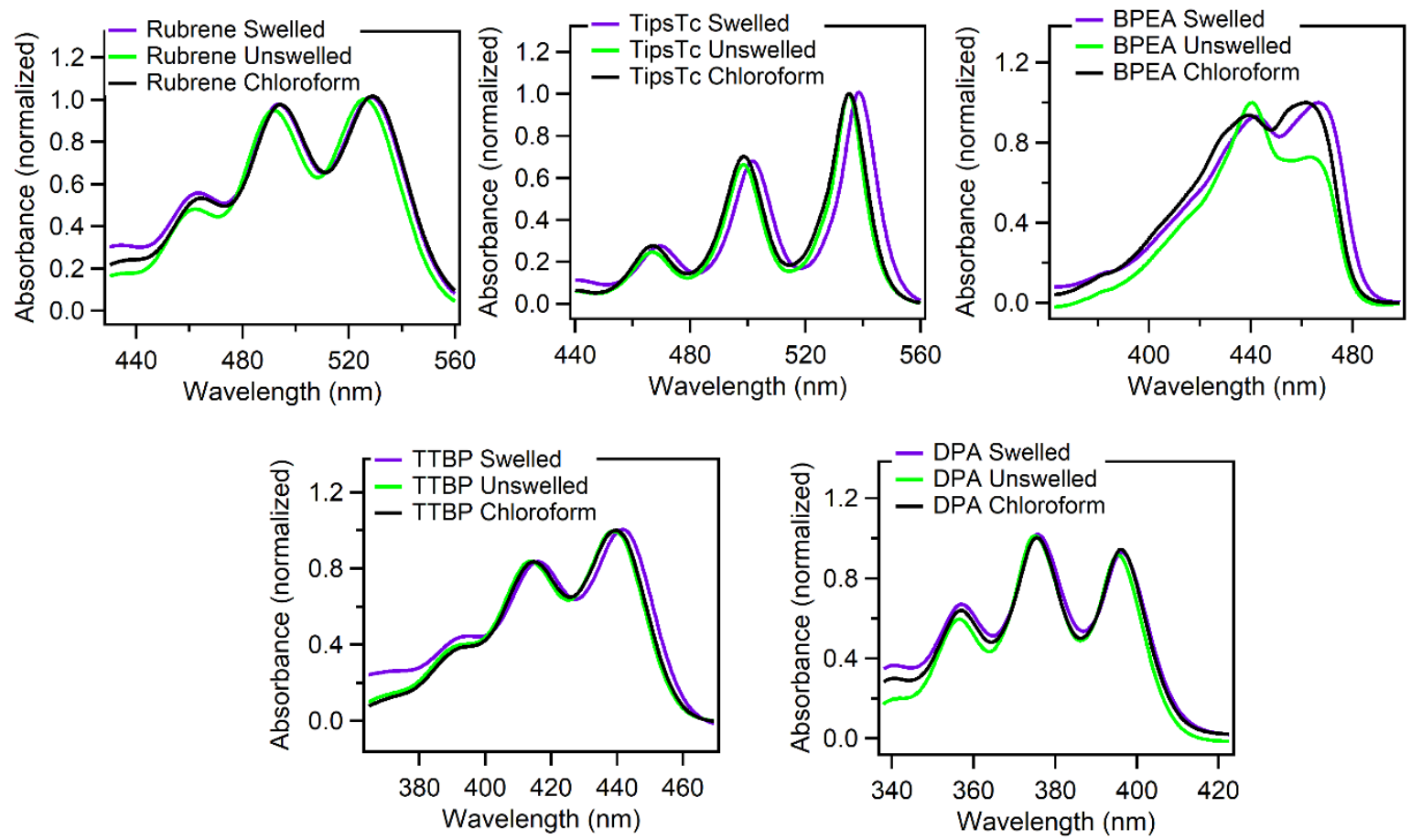

Figure S1. Absorption spectra for the annihilator component of swelled and unswelled micelles in water, as described in the main text, as well as a spectrum of annihilator as a dilute solution in chloroform for reference.

However, overall, perhaps the most striking result from these absorption spectra is the similarity between the annihilator spectra in the swelled and unswelled micelles. This result stands in contrast to differences in the fluorescence spectra, which are normalized and shown below in Figure S2. In that case, the fluorescence spectra from TipsTc, Rubrene and BPEA samples all reveal significant evidence for annihilator aggregation in the unswelled micelle case. This discrepancy occurs because fluorescence is a more sensitive probe of the molecular environment. Absorption occurs from a vertical geometry and nearly instantaneously, while fluorescence occurs over significantly longer periods of time (nanoseconds) after the excitation has had time to equilibrate in its local environment. Therefore, fluorescence can occur preferentially from lower energy states in the ensemble if they are available and is a more sensitive probe of them. 

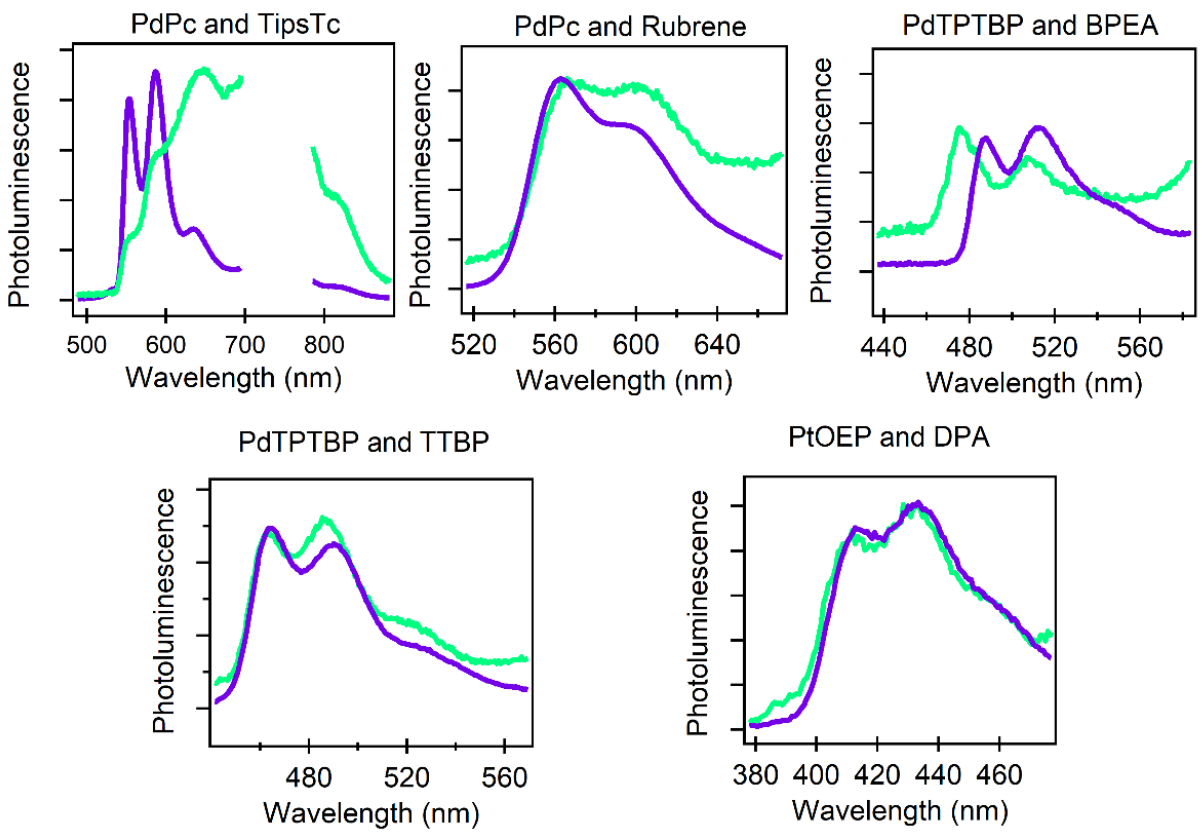

Figure S2. Normalized upconverted fluorescence spectra from swelled (purple) and unswelled (green) micelles. The relative counts are reported in the main text.

\section{Micelle Size}

Dynamic light scattering was performed on the swelled micelles, with the results presented in Figure S3. The average particle diameter (cumulants mean) is $48 \mathrm{~nm}$, which is significantly larger than the unswelled cumulants mean diameter reported for F127 micelles in water in the literature of $\sim 21.4 \mathrm{~nm} .{ }^{3}$ This result suggests the swelling affects the morphology of the particles and results in a size increase.

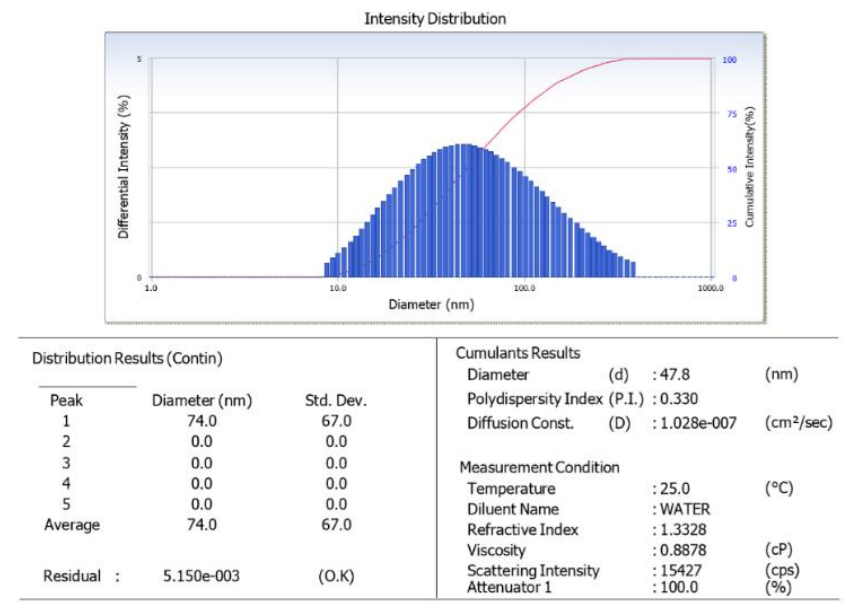

Figure S3. Dynamic light scattering on a typical swelled micelle composition reveals a singlet peak, centered around $48 \mathrm{~nm}$. 


\section{Transient Absorption Details and Fitting}

In Figure S4, we show example spectral and kinetic fits obtained by our global analysis procedure. For the example dataset, we have plotted the unswelled micelles with TipsTc and PdPc loaded into the core, excited at $730 \mathrm{~nm}$ as described in the main text. Using a simple fit where PdPc decays monoexponentially, concurrent with rise of the TipsTc signal and subsequent monoexponential decay, we are able to produce a satisfactory fit to the data. More details are provided below in Figure S5.
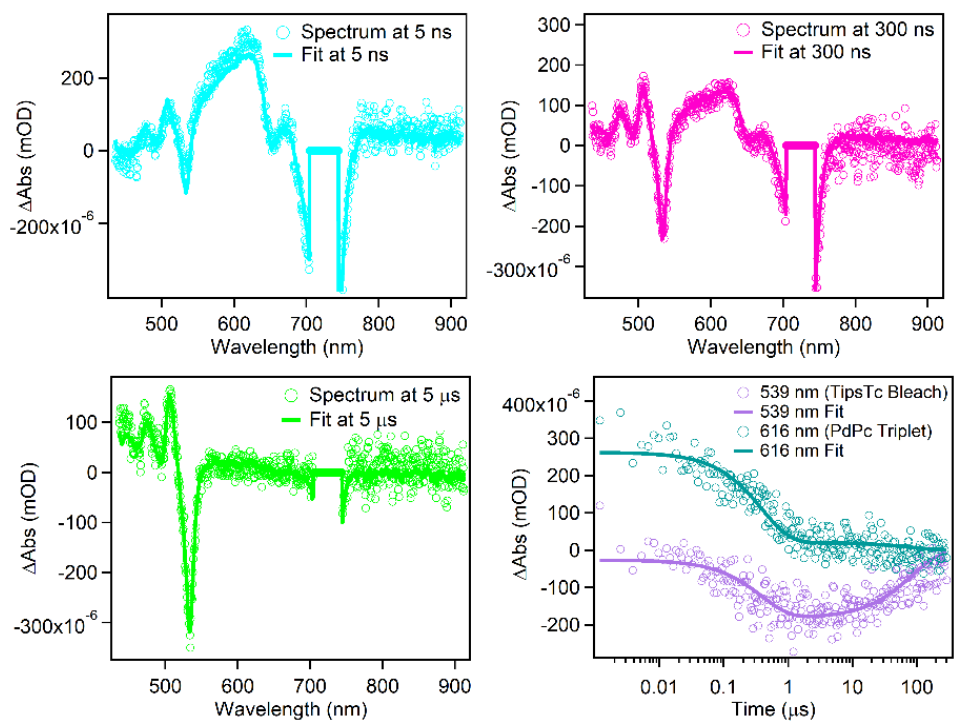

Figure S4. Comparison of data (open circles) and fits (solid lines) for selected spectral and kinetic slices. The dataset is from the unswelled TipsTc and PdPc micelles, excited at $730 \mathrm{~nm}$ as described in the main text.

In Figure S5, we provide further details of the global analysis. Specifically, for the unswelled upconversion micelles discussed in the main text, we reproduce the raw data color plot and compare it to the global analysis model. As seen in the residual plot, the global analysis is a satisfactory reproduction of the data with minimal residuals, using only two constant basis spectra, whose kinetics and spectra are shown in parts d) and e). We also show raw spectra at 30 ns and $7 \mu$ s where the spectra are dominated by the first and second basis function, respectively, showing the similarity of the data to the basis spectra. It is worth noting that for this particular system, the unswelled TipsTc and PdPc micelles, there is some TipsTc ground state bleach even in the earliest spectral component, despite excitation at $730 \mathrm{~nm}$ where TipsTc does not absorb. This suggests some fast transfer from PdPc to TipsTc in the absence of swelling that is not resolved in this measurement, consistent with more intimate contact and even some aggregation in the unswelled case, as discussed in the main text. 


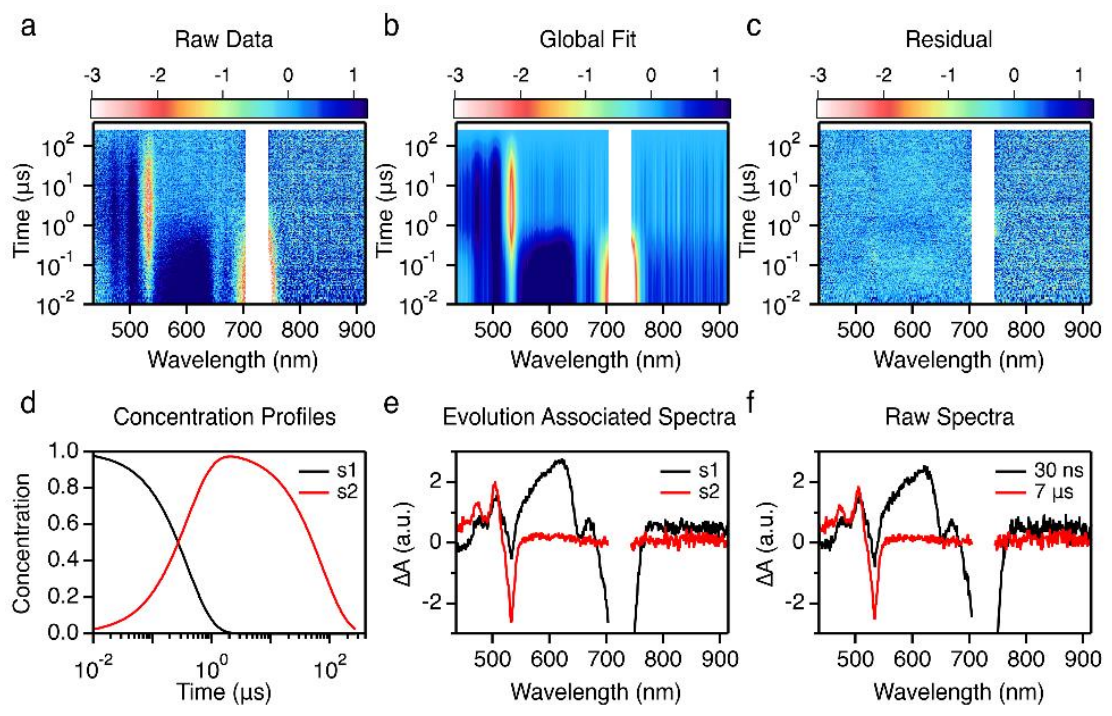

Figure S5. Comparison of a) the raw data for unswelled upconversion micelles containing TipsTc and PdPc to b) the global fit reconstruction of the dataset. In c) we show the residual plot, the subtraction of the global fit from the raw data, showing values near 0 and a satisfactory reproduction of the data. In d) we show the evolution of the populations as a function of time, where the two basis spectra s1 and s2 are shown in e). In f), we show raw spectra at 30 ns (when almost all population is in s1) and at $7 \mu$ s when the population is dominated by $s 2$.

\section{Amount of TCB Swelling}

Shown below, we investigated the effects of varying the TCB swelling agent loading during the micelle preparation. As the amount of TCB is increased, the resulting micelles produce more photoluminescence under identical $730 \mathrm{~nm}$ excitation conditions in monotonic fashion. However, even at $5 \mu \mathrm{L}$ of TCB, the spectrum is shifted from the unswelled case to closely resemble that of isolated TipsTc emission. Still, additional TCB results in more photoluminescence. However, after $20 \mu \mathrm{L}$ of TCB, the micelle size approaches that of visible light, causing scattering, as discussed below, so we limited our study to this loading. This result suggests the opportunity for even further improved upconversion characteristics if a better solvent can be found, or a system that perm its further swelling without causing scatter. 


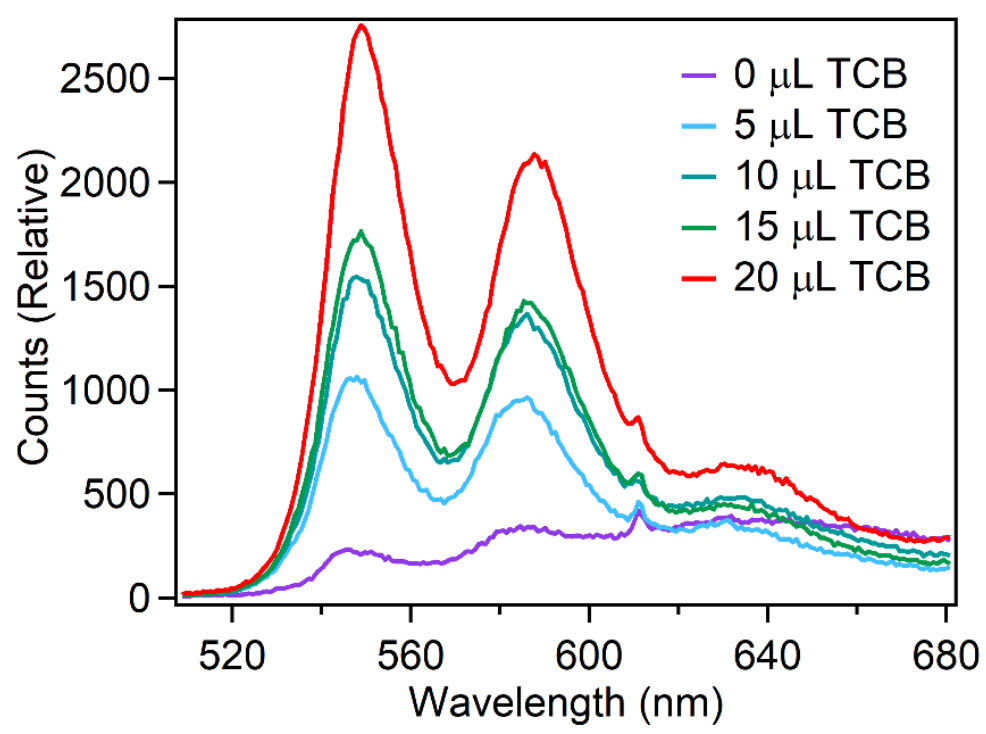

Figure S6. Relative counts for TipsTc and PdPc micelles measured under identical conditions (730 nm excitation) but prepared using variable amounts of TCB swelling agent during the preparation.

In Figure S7, we show the effect of increasing TCB content during micelle preparation on the optical properties of the resulting solutions. As micelles size increases to approach the size of visible light wavelengths, they cause scatter. However, filtration with a $0.45 \mu \mathrm{m}$ filter, as described in the micelle preparation protocol, removes the minority of micelles with sizes in this range and retrieves optically clear solutions, shown below. However, we limited our study to $20 \mu \mathrm{L}$ of TCB loading to prevent a significant fraction of our micelles from needing removal by filtration. After filtration, the clear solutions are stable and remain clear.

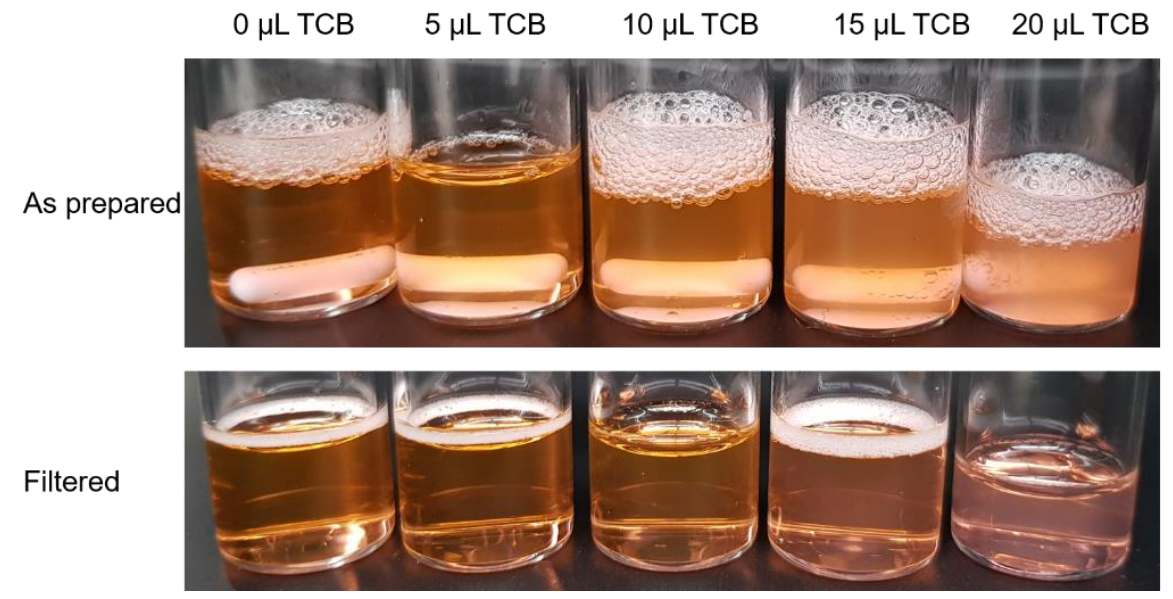

Figure S7. Photographs of micelle preparations with varying trichlorobenzene content in the preparation before and after passing through a $0.45 \mu \mathrm{m}$ poly vinylidene difluoride filter. 


\section{Stability}

In order to determine the stability of the micelles, we tested the swelled micelles freshly prepared, as well as a sample prepared and then aged four days. As shown below, there is not a significant change in upconversion properties, at least on the several day timescale.

TipsTc and PdPc Swelled Micelles

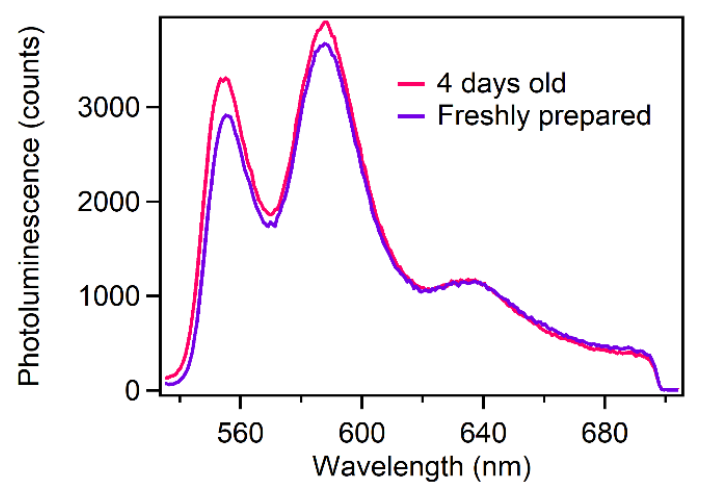

Figure S8. Upconversion under $730 \mathrm{~nm}$ excitation, detected with a $700 \mathrm{~nm}$ short pass filter, for a freshly prepared, swelled TipsTc and PdPc micelle solution, compared to a solution prepared and left four days before measurement.

\section{Biological Media}

To test the generalizability of this preparation to more biologically relevant media, such as solvents with some ionic content, we repeated our preparation but used Hanks' Balanced Salt solution from Gibco (containing a variety of salts, but predominantly 8 grams per liter of $\mathrm{NaCl}$ ) instead of water. The preparation proceeds similarly, and the upconversion photoluminescence of the resulting swelled TipsTc and PdPc micelles are shown below, comparing the two different media. We observe a small enhancement in photon upconverted luminescence for the salt solution micelles under identical illumination and detection conditions. This enhancement is modest but probably significant, and worthy of future investigation. Overall, the micelle performance is retained even in ionic environments more similar to those encountered in biological contexts.

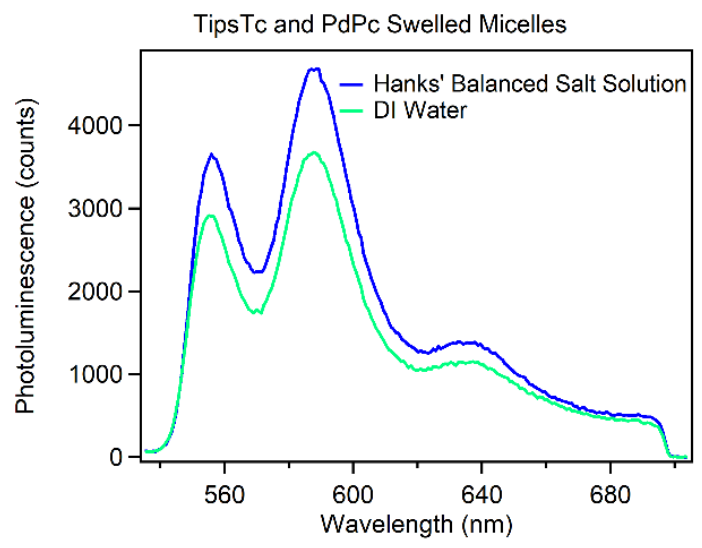

Figure S9. Comparison of upconverted photoluminescence (detected through a $700 \mathrm{~nm}$ short pass filter) for TipsTc and PdPc swelled micelles prepared in DI water as the medium (the typical preparation) versus using Hanks' Balanced Salt solution as the medium. 


\section{Notes on Micelle Synthesis}

Below we reproduce the micelle synthesis from the main text:

"To construct the upconverting micelles, we first mix $120 \mathrm{mg}$ of F127 in two $\mathrm{mL}$ of water. To this clear solution, we add a mixture of $0.2 \mathrm{mg}$ of annihilator and $0.0011 \mathrm{mg}$ of sensitizer dissolved in $30 \mu \mathrm{L}$ of chloroform and $20 \mu \mathrm{L}$ of 1,2,4 trichlorobenzene (TCB). This mixture is stirred vigorously (800 rpm) in a $7 \mathrm{~mL}$ vial where it becomes opaque. This opaque solution is continually stirred while uncapped in an inert atmosphere to evaporate off the chloroform, causing it to clarify $(\sim 16$ hours). After clarifying, this solution is passed through a $0.45 \mu \mathrm{m}$ poly vinylidene difluoride filter to produce the final, highly transparent, aqueous upconversion solution."

It is important to note that this procedure relies on evaporation of chloroform from an open vial. This process can be completed in different amounts of time based on vial size, stirring rate, etc. We have confirmed that the procedure works in the same fashion for $2 \mathrm{~mL}$ of total volume of water up to $20 \mathrm{~mL}$ of total volume. When running the procedure for the first time, if using a different scale or stir rate than that reported here, it is recommended to observe the solution as evaporation occurs. Shortly after addition of all components, large particles cause the solution to scatter light intensely and become opaque. When the vial has become fully clear, the final clear micelle solution is ready for use. Further evaporation after the solution becomes clear is not necessary, but is not detrimental either. Furthermore, it should be noted that in the absence of TCB, the procedure is completed on a much faster timescale, typically under an hour. This faster process occurs because the vapor pressure of chloroform is much larger in a neat chloroform solution relative to a mixed chloroform:TCB solution due to Raoult's Law. ${ }^{4}$

\section{References}

(1) Snellenburg S. P.; Seger, R.; Mullen, K. M.; van Stokum, I. H. M., J. J. . L. Glotaran: A Java-Based Graphical User Interface for the R Package TIMP. J. Stat. Soft. 2012, 49 (3), $1-22$.

(2) Odom, S. A.; Parkin, S. R.; Anthony, J. E. Tetracene Derivatives as Potential Red Emitters for Organic LEDs. Org. Lett. 2003, 5 (23), 4245-4248.

(3) Huo, Q.; Liu, J.; Wang, L.-Q.; Jiang, Y.; Lambert, T. N.; Fang, E. A New Class of Silica Cross-Linked Micellar Core-Shell Nanoparticles. J. Am. Chem. Soc. 2006, 128 (19), 6447-6453.

(4) van't Hoff, J. XII. The Function of Osmotic Pressure in the Analogy between Solutions and Gases. London, Edinburgh, Dublin Philos. Mag. J. Sci. 1888, 26 (159), 81-105. 